\title{
The Generic Network Model - an ITU Approach for Interoperability
}

\author{
Terje Henriksen, Telenor R\&D, \\ PO box 83, Kjeller, Norway \\ tel: +47 63848682 , fax: +47 63810076 , \\ email: terje-fredrik.henriksen@telenor.com
}

\begin{abstract}
Network interoperability may be achieved by a number of different means. This paper addresses the usage of management mechanisms to realize this goal. Where interoperability by means of management systems is concerned, standardization bodies like ITU are frequently associated with standardized network element (NE) models. This paper presents another option, interoperability on the network level. The network level view is more abstract and comprises fewer details, simplifying many management tasks considerably. A natural consequence of the proposal is the relaxation of the requirements for standardized NE models. The generic network model defined in the G.85x series is the core element of the proposal. The functionality of the current version as well as the planned extensions (access networks, connection-less networks including IP) are described and so are the underlying mechanisms, the generic network architecture defined in G.805, the Logical Layered Architecture in M.3010 and the enhanced Reference Model for Open Distributed Processing (RM-ODP) in G.851.1.
\end{abstract}

Keywords: Generic network architecture, generic network model, interoperability, interworking, logical layered architecture, management architecture, network level management, RM-ODP.

\section{Introduction}

The proposal made in this paper is for telecom network interoperability by means of management systems to be addressed on the network level rather than the network element level, for three reasons:

1. A widely accepted generic network architecture, the ITU-T rec. G.805 "Generic Functional Architecture of Transport Networks", exists already.

2. Interoperability at the network level is easier to achieve because the network view is more abstract and hence comprises fewer details. 
3. Focus 1 for the management of network technologies should be be shifted from the network element level to the network level anyway, to reduce complexity and thus, operational expenses.

A generic network level model is a prerequisite for the application of this approach. Such a model is being developed in Question 18 of Study Group 4 within ITU-T, in brief, Q.18/4. After a review of the generic network architecture and the Logical Layered Architecture this paper explains the applicability of the enhanced RM-ODP (Reference Model for Open Distributed Processing) framework to the development of the generic network model. The creation of a networkTTP (Trail Termination Point) is used as a modeling example throughout the RM-ODP viewpoints.

Multiple network technologies from multiple vendors spanning multiple network provider domains may interoperate following the approach outlined in this paper.

The existing model is applicable to connection-oriented core network technologies like SDH, WDM and ATM. Work has started in Q.18/4 to expand the scope by including connectionless technologies such as IP as well as access network technologies.

\section{The G.805 Generic Network Architecture}

In the early nineties, influenced by the emerging object oriented methodology, SG XVIII (later SG13) in ITU developed the generic network architecture [1]. It provides a high level view of the generic network function based on a small set of architectural entities (functional blocks) interconnected via reference points, see Fig.1. Two main network representations exist:

- Topology ${ }^{2}$ in terms of links, subnetworks and access groups.

- Connectivity in terms of trails, link connections, subnetwork connections, ports and reference points.

In an implementation, a visible reference point becomes an interface.

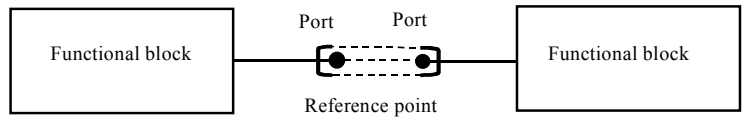

Fig. 1. Functional architecture, basic elements

The topological view describes the geographical distribution of the resources of a layer network. The access group is a container for a number of co-located access points. The subnetwork represents the routing capabilities within a site or grouping of sites and the link represents the transport capacity between subnetworks. Layering is a methodology for splitting the overall transport function into a hierarchy of layer networks on top of each other, each of which utilizing the service from the server layer to provide its own service. A topological view of a network consisting of three sites is shown in Fig.2.

\footnotetext{
${ }^{1}$ From the point of view of the network operator

${ }^{2}$ of a layer network
} 
The topological view is well suited when searching for candidate routes for connections. By introducing selection criteria such as the number of hops, the average spare capacity of the links, etc. in addition to the topological information, the search process may be automated.

All the architectural entities are available (but not necessarily visible) in the connectivity view, i.e., the ports, the reference points, the link connections, the subnetwork connections and trails as well as the topological entities.

The trail preserves the integrity of the information transported between access points at the boundary of the layer network by adding overhead at the transmit end and extracting and analyzing it at the receive end.

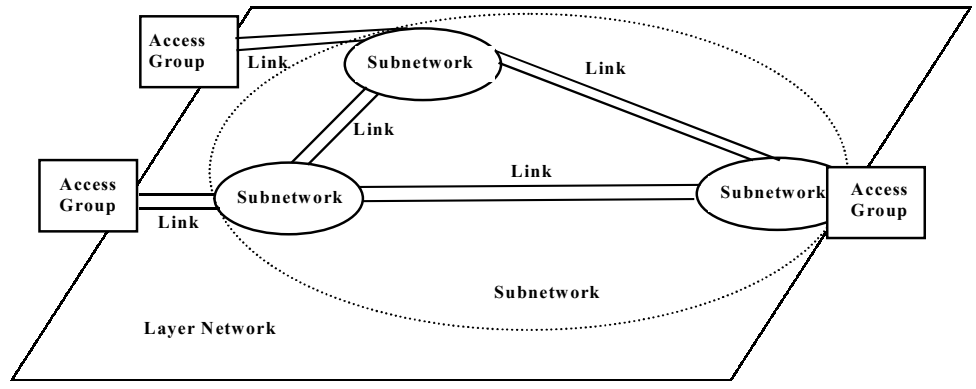

Fig. 2. Network architecture - the topological view



Fig. 3. The connectivity view - client and server layer networks

The subnetwork and the associated subnetwork connections provide the capability of flexibly routing the incoming traffic of a site to the appropriate outgoing links. The routing control may be realized by management or signaling. 
The interlayer relationship is provided by the adaptation function as shown in Fig.3. The capability of individual management for each layer is a general requirement. To make this possible, the link connections are there to provide a virtual representation in the client layer of the server layer support.

Partitioning of subnetworks implies that a composite subnetwork has an internal structure so that it may be recursively decomposed into component subnetworks interconnected by links. An example of subnetwork partitioning is illustrated in Fig.2. The outer subnetwork is a composite subnetwork comprising three component subnetworks.

In a similar fashion, subnetwork connections may be partitioned into component subnetwork connections interconnected by link connections. Partitioning of subnetworks and subnetwork connections may be utilized to represent parts of a layer network at different levels of abstraction.

The approach taken when developing G.805 is generic in the sense that it is not referencing any particular technology. Most of the architectural entities defined are applicable to a broad range of technologies, eventually with the behavior slightly modified. At the moment, the following network architectures exist:

- G.803 - SDH [2]

- G.872 - WDM [3]

- I.326 - ATM [4]

- G.902 - Access networks [5]

- g.cls - Connectionless communication [6]

The last one, g.cls, is currently under development. It will cover connectionless technologies such as IP.

\section{The Logical Layered Architecture - Relaxed Requirements for Standardised Ne-Models}

The Logical Layered Architecture in Fig.4 is part of the TMN but has been applied in a number of non-TMN applications as well.

${ }^{3}$ The actual server layer support is the sequence of the adaptation function, the server layer trail and the other adaptation function in Fig.3. 


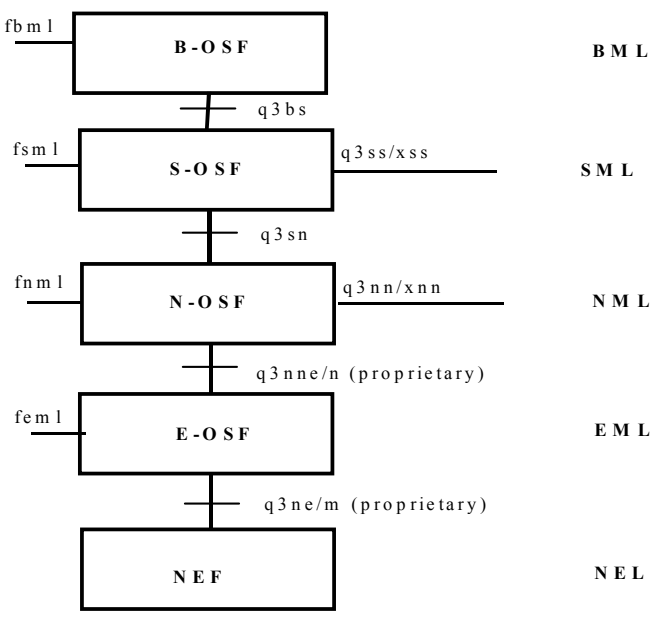

Fig. 4. The Logical Layered Architecture

As with the network architecture, the LLA consists of functional blocks bound together via standardized $(\mathrm{q}, \mathrm{x}, \mathrm{f})$ or proprietary ${ }^{4}(\mathrm{~m}, \mathrm{n})$ reference points. On top of the basic information transport function, the Network Element Function (NEF), there is a hierarchy of management layer $\$ 5$ providing management functions (OSFs) in a layered fashion, i.e., the functions at one layer rely on the service of its server layer to perform its own service. The layers are frequently referred to as different levels of management abstraction, the higher the layer, the higher the level of abstraction.

The Element Management Layer (EML) is performing management operations pertaining to each individual NE. No knowledge of the relationship with other NEs is presumed.

The execution of an operation on the EML may result in interactions between the EML and the NEF across the $\mathrm{q} 3 \mathrm{ne} / \mathrm{m}$ reference point. The protocol and model governing these interactions are often proprietary as they depend on equipment manufacturer properties to a large extent.

Network level operations like setting up connections are carried out at the Network Management Layer (NML). As a result, connection commands are sent to the EML across the appropriate q3nne reference points based on the topological knowledge of the network structure held by the NML. These operations are, however, normally not visible across the q3nn or q3sn reference points.

The Service Management Layer (SML) is managing services running on top of the NML.

The Business Management Layer is managing business processes within the organization of the network provider and, in some cases, his customers.

Management systems may be recursively nested within the NML and the SML. They may also interoperate with other management systems within the same administrative domain (same network provider) across q3nn or q3ss reference points,

${ }^{4}$ Strictly speaking, proprietary reference points are not part of the LLA.

${ }^{5}$ One may also speak about a certain level, such as the network management level. 
or in other administrative domains across xnn or xss reference points. The f-reference point for operator access may be provided in all layers.

Network technologies may be combined to provide more comprehensive functions. Of particular interest is the case where IP is running on top of different access and core network technologies to provide a unified end-to- end network representation to all services in the SML. This is the so-called "Full Service Network" (FSN) concept. The access portion of the FSN is often denoted the "Full Service Access Network" (FSAN).

In principle, every management task should be handled at the highest possible level of abstraction because, potentially, that makes the task simpler. For example, instead of setting up a connection at the NML, the connections in each node may be set up individually at the EML but that takes more time.

When an alarm occurs in an NEF it is detected by the EML and mapped to the NML so that the faulty network connections are identified. Depending on the importance of the traffic and the protection and restoration mechanisms available the traffic may or may not be rerouted by the NML. The task of repairing the fault is left to the EML - when time and priority permits.

The other usage of the EML is for the configuration of the NEF during installation or upgrade. There seems to be a trend towards limiting operator intervention to the setting of configuration options that may neither be done in the factory nor automated.

A consequence of dealing with interoperability at the NML is that the requirements for standardized NE models at the EML may be relaxed, i.e., the q3nne and q3ne reference points may be replaced by proprietary $\mathrm{n}$ and $\mathrm{m}$ reference points. To allow the parties involved (the management system manufacturer, the network provider, third party implementers) to perform the implementation in a controlled and orderly manner, however, $\mathrm{n}$ and $\mathrm{m}$ must be well specified. Taken together with increased automation of EML functions wherever possible, reduction in complexity and thus in operational expenses should result.

If interoperability is more attractive on the NML what about even higher layers, i.e., the SML? Up to now, standardizing SML models have been regarded as interfering with proprietary service implementations. Certain initiatives have been provided such as the standardization of the leased line and the BVPN services in the ITU and the definition of the service management architecture in the TMF [8]. This may change with the forecasted extensive deployment of new services such as ecommerce on top of the IP network.

\section{Modelling Methodology - the Enhanced RM-ODP Framework}

Due to a number of limitations, in particular the absence of mappings to management requirements and the lack of support for distribution, Q.18/4 concluded that OSI Management did not have sufficient modeling capabilities to become the modeling methodology of choice for the development of the network model. Instead, it was decided that an enhanced version of the Reference Model for Open Distributed Processing (RM-ODP) should be developed. The resulting methodology [9], [10] and 
the model for an example, subnetwork connection management [11], [12], [13] were developed. They became approved in 1996.

RM-ODP is defined in [14], [15], [16] and [17]. It consists of five viewpoints:

- The Enterprise Viewpoint

- The Information Viewpoint

- The Computational Viewpoint

- The Engineering Viewpoint

- The Technology Viewpoint

Each viewpoint is a self-contained specification of the system from a particular perspective. In addition, certain mappings between the viewpoints need to be maintained for the integrity of the overall system to be preserved.

Because the target is a model for management, only the system aspects subject to management, the management requirements, need to be modeled. They are expressed as actions with associated policies in the Enterprise Viewpoint. This implies that the requirements become an integrated part of the model.

\subsection{The Enterprise Viewpoint}

When compared with the original version of RM-ODP, most of the changes pertain to the Enterprise Viewpoint. A finer modeling granularity is allowed to correspond with the granularity of the network resource types in G.805. All the constructs (in all viewpoints) are provided with unique labels for backward traceability from the model elements in the Information-, Computational-, and Engineering Viewpoints to the functional requirements in the Enterprise Viewpoint. This is a fundamental mechanism for the support of conformance testing and also when estimating the cost of implementation for particular requirements.

Functional requirements are modeled as actions invoked by a caller role ${ }^{6}$ and carried out by a provider role. Actions are enforced or restricted by action policies, Permissions, Prohibitions or Obligations respectively, to provide additional level of detail to the action definition.

Actions are grouped into functional units called Communities. An ordered series of actions combined to provide more comprehensive functions is called an Activity. By taking advantage of existing communities, substantial reuse of specification and eventually implementation may be achieved.

The Service Contract is essentially a listing of the functional contents of a community. Due to the labels it provides unique references to the functional elements already defined, simplifying reuse. The service contract may be used to define the technical part of the Service Level Agreement (SLA), the Service Level Specification (SLS).

In addition to capturing the functional requirements, the Enterprise Viewpoint also serves as the roadmap towards the other viewpoints. Actions in the Enterprise Viewpoint map to interface operations in the Computational Viewpoint. The client and provider roles map to computational objects. Enterprise actions are normally concerned with the manipulation (create, delete, associate, etc.) of G.805 network

\footnotetext{
${ }^{6}$ in RM-ODP, a role is a fraction of the behavior of an object, in this case, an Enterprise object
} 
resources such as trails, access groups etc. Network resources map to objects, attributes or relationships in the Information Viewpoint.

When passing from the less formal architectural description of network resources in G.805 to a formal network model, additional behavior needs to be settled. Rec. G.852.2 "Transport Network Enterprise Model [18] is doing that for the elements already defined in G.805 and is also providing definitions for some important elements currently missing.

An extract from the Enterprise Viewpoint for trail management showing the role, action and service contract definitions for trail termination point creation is provided below.

\section{Community trail management}

\section{Role}

\section{tm_caller}

This role reflects the client of the actions defined within this community. One and only one caller role occurrence must exist in the community.

\section{tm_provider}

This role reflects the server of the actions defined within this community. One and only one provider role occurrence must exist in the community.

\section{layer network domain}

This role represents the layer network domain resource defined in Recommendation G.852.2. One and only one layer network domain role occurrence may exist in the community.

\section{trail termination point}

This role reflects the trail termination point resource as defined in Recommendation G.852.2. Zero or more trail termination point role occurrences may exist within this community.

\section{ACTION}

\section{Create trail termination point}

This action is used for the creation of a trail termination point. The caller has the ability to provide a unique user identifier to identify the trail termination point that has been created.

\section{ACTION_POLICY}

OBLIGATION inputDirectionality

The caller shall specify the directionality of the trail termination point to be created.

PERMISSION inputUserId

The caller may provide a user identifier for the requested trail termination point.

OBLIGATION rejectUserIdNotUnique

If PERMISSION inputUserId is part of the contracted service and if the user identifier is not unique in the provider context, then the provider shall reject the action.

OBLIGATION provideUserId

If PERMISSION inputUserId is part of the contracted service, then the provider shall use the user identifier as the unique identifier when communicating with the caller.

OBLIGATION successReturnId 
If PERMISSION inputUserId is not part of the contracted service, the provider shall, upon success of this action, return the unique identifier for the created trail termination point.

PERMISSION inputUserLabel

The caller may provide a user label for the requested trail termination point.

\section{SERVICE CONTRACT tm_src \\ ROLE}

tm_caller, tm_provider, layer network domain, trail termination point

ACTION

Create trail termination point \{OBLIGATION inputDirectionality, rejectUserIdNotUnique, provideUserId, successReturnId; PERMISSION inputUserId\};

\subsection{The Information Viewpoint}

The Information Viewpoint describes the static behavior of the system in terms of information elements, i.e., objects, attributes and relationships. The behavior of the information objects is made up of invariants, attributes and mandatory relationships. It may either be provided as structured English text or be described using the formal language $\mathrm{Z}$ [19].

Rec. G.853.1 "Common Information Viewpoint"[20] is a library constituting the information elements that may be directly defined on the basis of G.805 and G.852.2, that is, without taking functional requirements pertaining to any specific management area into account. When producing an Information Viewpoint specification for a specific functional area the elements from the library are being used as superclasses and specializations are provided taking the additional functional requirements into account. New elements may be added as well.

Information elements are mapped to the corresponding Enterprise Viewpoint elements, eventually to elements in G.852.2.

The Information Viewpoint is the ultimate source for the definition of the information elements within the system. This is reflected by the Parameter Matching clause in the Computational Viewpoint which maps the input and output parameters to the corresponding information elements.

An extract from the Information Viewpoint for trail management showing the UML class diagrams, the information objects and the relationships for networkTTP creation is shown below. 




Relationship diagram

Inheritance diagram

Fig. 5. UML class diagrams for trail termination point creation

\section{tmNetworkTTP}

$<$ COMMUNITY: trail management, ROLE: trail termination point $>$ DEFINITION

"This object class is derived from $<$ networkTTP $>$."

ATTRIBUTE

$<$ userLabel $>$

"<COMMUNITY: trail management, ACTION: create trail termination point, ACTION_POLICY: inputUserLabel>

This attribute is imported from G.853.1 and is used as a user friendly label for the networkTTP."

\section{RELATIONSHIP}

$<$ accessGroupIsMadeOfNetworkTTPs $>$

$<$ trailIsTerminatedByPointToPoint $>$

$<$ subnetworkTPIsRelatedToExtremity $>$

$<$ layerNetworkDomainIsMadeOf $>$

\section{tmLayerNetworkDomain}

$<$ COMMUNITY: trail management, COMMUNITY_POLICY: signalId $>$ DEFINITION

"This object class is derived from < layerNetworkDomain>."

\section{ATTRIBUTE}

-- none additional

RELATIONSHIP

$<$ layerNetworkDomainIsMadeOf $>$ 


\section{layerNetworkDomainIsMadeOf (imported from G.853.1)}

DEFINITION

"The layerNetworkDomainIsMadeOf relationship class describes the relationship that exists between a layerNetworkDomain and the objects that compose it."

ROLE

containerLND

"Played by an instance of the <layerNetworkDomain> information object type or subtype."

element

"Played by an instance of the subtype of the <networkInformationTop $>$ information

object type."

INVARIANT

inv_containerLNDRoleCardinality

"One and only one instance of the role containerLND must participate in the relationship."

inv_elementLNDRoleCardinality

"One or more instances of the role element must participate in the relationship." inv signalIdentification

"The containerLND and the element must contain the same signalIdentification information."

\subsection{The Computational Viewpoint}

The dynamic system behavior is described as interactions between computational objects in the Computational Viewpoint. Operational as well as notification interfaces are provided by these objects. The computational objects represent the finest granularity of objects in the mapping 7 to the Engineering Viewpoint.

For each computational object, the mapping is provided to the appropriate caller and provider roles in the Enterprise Viewpoint.

The major part of the Computational Viewpoint deals with the specification of the operations belonging to each interface. The methodology used is commonly known as "Design by contract" [21]. An operation is invoked providing a number of input parameters and upon the successful execution, a number of output parameters are returned. Each parameter has a name, a type specifier and a value assigned. Every parameter is mapped to the corresponding element in the Information Viewpoint in the Parameter Matching clause.

The invariant state of the system before and after the execution of an operation is defined by a set of pre- and post-conditions. They are defined by the state of the relevant relationships and attributes. Whenever an invariant is violated, a specific exception is raised. For each exception, an explanatory text as well as a type specifier is provided.

A report notification is defined for every operation. The report may contain additional information for the recipients to take advantage of the outcome of the successful event.

\footnotetext{
${ }^{7}$ This is a formal mapping defined in RM-ODP
} 
The operations are described in a communication protocol neutral fashion. Protocol specific constructs for the communication protocol chosen are added in the Engineering Viewpoint. The parameters are defined in the ASN.1 language.

Operations are mapped to actions in the Enterprise Viewpoint.

An extract from the Computational Viewpoint for trail management describing the create networkTTP operation and the associated ASN.1 types, is shown below.

\section{Create networkTTP}

$<$ COMMUNITY: trail management, ACTION: create trail termination point> OPERATION createNetworkTTP \{

INPUT PARAMETERS

layerND: LayerNetworkDomainChoice;

pointDir: PointDirectionality;

suppliedUserIdentifier: UserIdentifier;

-- zero length string or 0 implies none supplied.

suppliedUserLabel: GraphicString;

-- zero length implies none supplied.

\section{OUTPUT_PARAMETERS}

networkTTP: NetworkTTPChoice;

\section{RAISED_EXCEPTIONS}

userIdentifierNotUnique: UserIdentifier;

failureToCreateNetworkTTP: NULL;

failureToSetUserIdentifier:NULL;

BEHAVIOUR

SEMI_FORMAL

PARAMETER_MATCHING

layerND: INFORMATION OBJECT: tmLayerNetworkDomain>;

suppliedUserIdentifier: <INFORMATION ATTRIBUTE: resourceId >;

pointDir: <INFORMATION ATTRIBUTE: pointDirectionality>;

networkTTP: <INFORMATION OBJECT: tmNetworkTTP>;

suppliedUserLabel: <INFORMATION ATTRIBUTE: userLabel>;

\section{PRE CONDITIONS}

inv_uniqueUserIdentifier

"suppliedUserIdentifier shall not be equal to resourceId of any element in a <layerNetworkDomainIsMadeOf> relationship where layerND refers to containerLND."

- POST_CONDITIONS

- inv_existingNetworkTTP

- "networkTTP and layerND must respectively refer to element and containerLND in a <layerNetworkDomainIsMadeOf> relationship."

- inv_agreedUserIdentifier

"resourceId of tmNetworkTTP referenced by networkTTP is equal to

suppliedUserIdentifier, if it is supplied." 


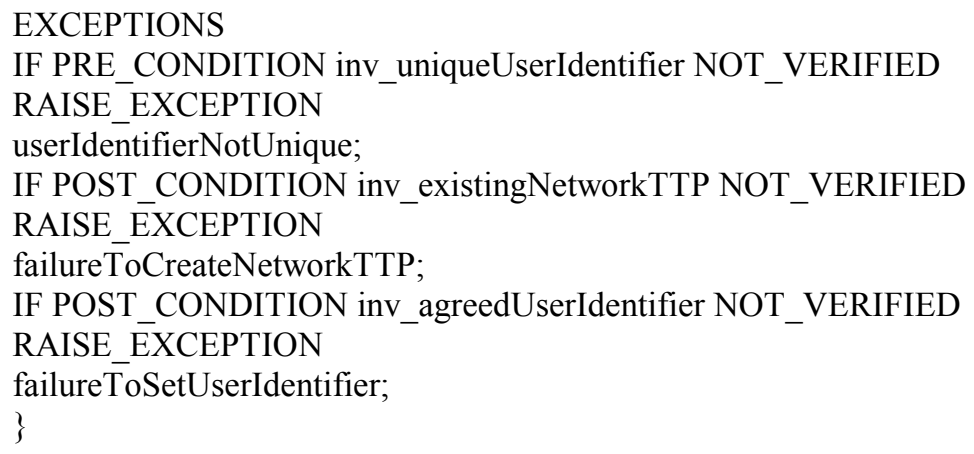

\section{Supporting ASN.1 productions}

LayerNetworkDomainChoice ::= CHOICE \{

tmLayerNetworkDomainQueryIfce TmLayerNetworkDomainQueryIfce, userIdentifier UserIdentifier \};

NetworkTTPChoice::= CHOICE \{ tmNetworkTTPQueryIfce TmNetworkTTPQueryIfce, userIdentifier UserIdentifier\};

\subsection{The Engineering Viewpoint}

The Engineering Viewpoint describes the operations for specific interfaces based on a given communication protocol. Specifications exists already for CMIP [22] [23] and Corba communication [24], [25], [26] and others will be provided. When utilizing CMIP, Managed Object (MO) classes representing network resources are mapped to the Enterprise Viewpoint resources in G.852.2. Actions are mapped to operations in the Computational Viewpoint and name bindings and attributes are mapped to the corresponding elements in the Information Viewpoint. The mapping scheme for Corba communication is not fully decided yet.

Distribution is another important feature for future applications. Within RM-ODP, there are mechanisms included to implement a number of distribution types by supporting the corresponding transparencies.

The functionality describing the network TTP creation for a CMIP interface is spread across a number of constructs, the MO class definition, the conditional packages, the name binding and the error parameters. It is not readily separable from a number of other functional elements either and, therefore, will not be presented here.

\subsection{The Technology Viewpoint}

The Technology Viewpoint is concerned with implementation issues only and will not be discussed here. 


\section{Existing Functionality}

Following the approval of the enhanced RM-ODP framework in 1996, a range of recommendations applicable to technologies such as SDH, WDM and, to a certain extent ATM, were approved in 1999. The following functional areas were covered:

- Topology management [27], [28], [29].

- Pre-provisioned adaptation management [30], [31], [32].

- Pre-provisioned link connection management [33], [34], [35].

- Pre-provisioned link management [36], [37], [38].

- Trail management [39], [40], and [41].

Work is in progress to include partitioning, protection, routing, and failure propagation and also completing the model for ATM.

When assessing the applicability of a new technology to the model, the specific requirements of the technology are analyzed. In some cases the non-matching requirements may be modeled on the NE level, while others may lead to technology specific extensions on the network level. For example, the assessment of ATM resulted in two major changes, the redefinition of link capacity to be either bandwidth or the number of link connections, and the notion of dynamic creation of termination points during connection setup.

The applicability of the model to WDM technology was proven by the ACTS project Mephisto and demonstrated at the public presentation in October 1999 [42]. The setup of a protected trail in the $\mathrm{OCH}$ layer on a prototype system consisting of three OADMs in a ring structure was shown. The network model used in Mephisto is based on the generic network model. The configuration manager developed provides the operator with one operation for each action in the communities supported, that is, topology management, pre-provisioned link connection management, subnetwork connection management and trail management.

It is argued in the Mephisto project [43] that the generic model may be utilized for the management of SDH over WDM, provided that an enhanced version of the route discovery function is used in the $\mathrm{OCH}$ layer. An alternative solution to limit noise accumulation is the addition of a digital frame like the digital wrapper on top of the OCH layer combined with Forward Error Correction (FEC) inside each network provider domain involved.

So far, only technologies for the core network have been modeled. There are, however, increasing concerns to provide an end-to-end network view including access network technologies as well. A combined network and network element model for ATM PONs will be approved this year in ITU-T [44].

\section{Modelling Connectionless Communication}

The current versions of G.805 and the technology specific extensions all presume connection - oriented communication, that is, prior to traffic flowing, a connection has to exist. With connectionless communication there is no connection setup in advance. To cope with that, a novel network architecture with the working title "g.cls" is currently being defined in SG.13. The scope is not limited to connectionless 
technologies, so there is a potential for g.cls to become the replacement of G.805 rather than a complement. Developing a network model for connectionless technologies is part of the work plan for Q.18/4.

If IP is to become the major network technology of the future, enhancements in the routers as well as in the applications running in the hosts are necessary. A comprehensive network model is a powerful tool for analyzing as well as managing such an enhanced IP based network.

One of the major enhancements relates to the communication paradigm. It is widely agreed that the current "Best Effort" approach should not be the only level supported. Traffic Engineering is a tool that are being proposed to provide differentiation to the communication paradigm and thus to the QoS utilizing mechanisms such as RSVP [45], Intserv [46], [47], Diffserv [48], [49], MPLS [50], [51] and COPS [52], [53], [54]. In addition, a control framework is needed to verify compliance between planned and actual traffic and also for specifying how to deal with excess traffic. At the same time, one is trying to preserve the structural simplicity of the existing Internet.

Differentiated service quality should lead to differentiated charging. Consequently, new charging models must be developed to complement the existing flat rate charging.

The second argument for an extension of the model is the interworking between IP and other technologies. Primarily, client-server schemes with IP in the client role are foreseen, but peer configurations are expected too. A variety of interworking schemes need to be taken into account, ranging from gateways supporting one specific management function to the full interworking between IP and the server technologies.

Another concern is that of handling the vast forecasted increase in traffic [55]. This traffic will be aggregated and handled by large capacity routers. Unfortunately, data traffic on the one hand and voice and video traffic on the other poses quite different requirements to the network in terms of packet loss, delay and delay variation. This makes the traffic grooming function more complex. There are issues relating to the work split between routing on the client level (IP) and routing on the server level (OTN/WDM) too.

\section{A Multi-technology/MULTI-domain Example}

Two networks based on compatible technologies (for the traffic) are exchanging traffic across a domain boundary, confer Fig. 6.

\footnotetext{
${ }^{8}$ QoS should be interpreted broadly including every kind of parameter affecting the perception of the end user of the service provided.
} 


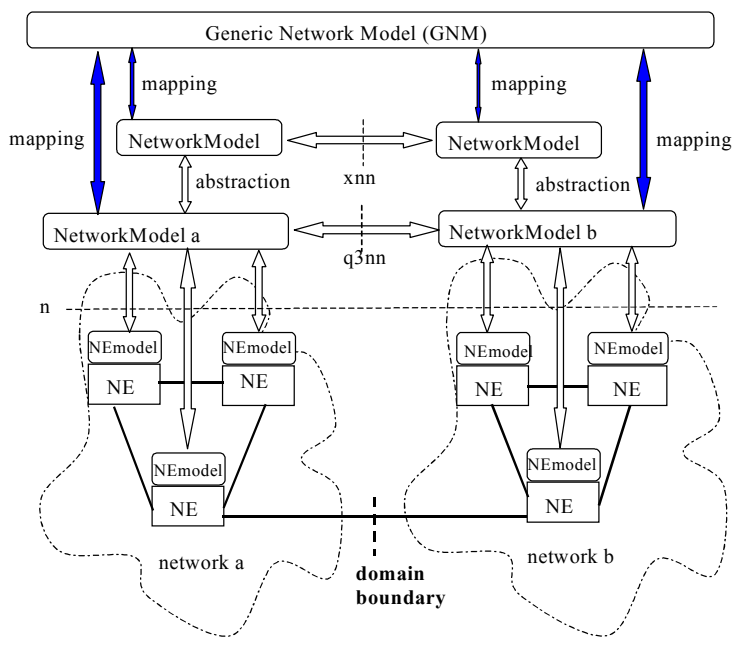

Fig. 6. Scenario for multi-technology, multi-domain interoperability

The network modeld 9 in both domains are based on the generic model but differences may exist due to manufacturer, equipment type/-version, technology specific properties, etc., catered for by the term "mapping" in Fig.6. The two domains may be operated by the same network provider, in which case there is a standardized q3nn reference point available for network level management across the domain boundary. With two different providers, there is a xnn reference point instead based on a more abstract network model.

The interactions between the network element models and the network models take place via proprietary $\mathrm{n}$ reference points. As long as the network models in the two domains are being consistently updated whenever changes occur on the NE level and vice versa, the capability of providing well defined end to end functions is maintained.

The generic network model (GNM) is providing the operators with an overall view of the networks in the two domains. On this basis the operators may find a route for an end-to-end trail and input a trail request to the GNM. The trail request is converted into SNC requests to the network models in the two domains (x or q) and further to cross connection requests to the appropriate NE models. Finally, crossconnections are established in the NEs to complete the setup of the end-to-end trail.

\section{Conclusion}

Interoperability is an attractive feature in a heterogeneous telecom network environment. A number of different approaches are being proposed for achieving that. This paper suggests that interoperability at the network level is a natural choice and

9 All the models in Fig.6 including the generic network model are physical models implemented in databases. 
proceeds by describing the underlying tools and the existing version of the generic network level model as defined in the G.85x series of recommendations.

The consequence of addressing interoperability at the network level is that the requirements for a standardized NE model become relaxed. Taken together with increased automation of NE functionality, substantial savings in operational expenses are expected.

Technologies like SDH, WDM and, partly, ATM are supported by the current version of the model. Work has started to expand the scope by including technologies for the access network and for connectionless communication such as IP. This will make the generic network model well suited for modeling end-to-end trails across multiple network provider domains and multiple technologies spanning the access network as well as the core network.

\section{References}

1. ITU-T rec. G.805 "Generic functional architecture of transport networks", Geneva 11/95

2. ITU-T rec. G.803 "Architecture of transport networks based on the synchronous digital hierarchy (SDH)", Geneva 06/97

3. ITU-T draft rec. G.872 "Architecture of optical transport networks", Geneva 02/99

4. ITU-T rec. I.326 "Functional architecture of transport networks based on ATM", Geneva $11 / 95$

5. ITU-T rec. G.902 "Framework recommendation on functional access networks (AN). Architecture and functions, access types, management and service node aspects", Geneva $11 / 95$

6. ITU-T draft. rec. g.cls "G.cls functional model”, Kyoto 03/00

7. ITU-T draft.rev.rec. M.3010 "Principles for a telecommunications management network architecture", Geneva, January 2000

8. TMF, Telecom Operations Map, GB910, version 1.1, April 1999

9. ITU-T rec. G.851.1 "Management of the transport network - application of the RM-ODP framework", Geneva, 03/99

10. Varma, E.L., et al "Achieving global information networking", Artec House, Boston/London, 1999

11. ITU-T rec. G.852.1 "Management of the transport network - Enterprise viewpoint for simple subnetwork connection management", Geneva, 11/96

12. ITU-T rec. G.853.2 "Subnetwork connection management information viewpoint", Geneva, $11 / 96$

13. ITU-T rec. G.854.1 "Management of the transport network - Computational interfaces for basic transport network model", Geneva, 11/96

14. ITU-T rec. X.901 "Basic reference model for Open Distributed Processing- Part 1: Overview"

15. ITU-T rec. X.902 "Basic reference model for Open Distributed Processing- Part2: Foundations"

16. ITU-T rec. X.903 "Basic reference model for Open Distributed Processing- Part 3: Architecture"

17. ITU-T rec. X.904 "Basic reference model for Open Distributed Processing- Part 4: Architectural Semantics"

18. ITU-T rec. G.852.2 "Management of transport network- Enterprise viewpoint description of transport network resource model", Geneva, 03/99

19. Potter, B. et al "An introduction to formal specification and Z", Prentice Hall, 1992 
20. ITU-T rec. G.853.1 "Management of transport network- Common elements of the information viewpoint for the management of a transport network", Geneva, 03/99

21. Meyer, B. "Object oriented software construction", Prentice Hall 1997

22. ITU-T rec. M.3100 amd.1 "Generic network information model Amendment 1", Geneva, $03 / 99$

23. ITU-T rec. G.855.1 "Management of transport network- GDMO engineering viewpoint for the generic network level model", Geneva, 03/99

24. ITU-T draft. rec. X.780 "TMN guidelines for defining CORBA Managed Objects", London, 05/00

25. ITU-T draft. rec. Q.816 "CORBA based TMN services", London, 05/00

26. ITU-T draft. rec. M.3120 "CORBA generic Network and NE level information model", London, 05/00

27. ITU-T rec. G.852.3 "Management of transport network- Enterprise viewpoint for topology management", Geneva, 03/99

28. ITU-T rec. G.853.3 "Management of transport network- Information viewpoint for topology management", Geneva, 03/99

29. ITU-T rec. G.854.3 "Management of transport network- Computational viewpoint for topology management", Geneva, 03/99

30. ITU-T rec. G.852.8 "Management of transport network- Enterprise viewpoint for preprovisioned adaptation management", Geneva, 03/99

31. ITU-T rec. G.853.8 "Management of transport network- Information viewpoint for preprovisioned adaptation management", Geneva, 03/99

32. ITU-T rec. G.854.8 "Management of transport network- Computational viewpoint for preprovisioned adaptation management", Geneva, 03/99

33. ITU-T rec. G.852.10 "Management of transport network- Enterprise viewpoint for preprovisioned link connection management", Geneva, 03/99

34. ITU-T rec. G.853.10 "Management of transport network- Information viewpoint for preprovisioned link connection management", Geneva, 03/99

35. ITU-T rec. G.854.10 "Management of transport network- Computational viewpoint for preprovisioned link connection management", Geneva, 03/99

36. ITU-T rec. G.852.12 "Management of transport network- Enterprise viewpoint for preprovisioned link management", Geneva, 03/99

37. ITU-T rec. G.853.12 "Management of transport network- Information viewpoint for preprovisioned link management", Geneva, 03/99

38. ITU-T rec. G.854.12 "Management of transport network- Computational viewpoint for preprovisioned link management", Geneva, 03/99

39. ITU-T rec. G.852.6 "Management of transport network- Enterprise viewpoint for trail management", Geneva, 03/99

40. ITU-T rec. G.853.6"Management of transport network- Information viewpoint for trail management", Geneva, 03/99

41. ITU-T rec. G.854.6 "Management of transport network- Computational viewpoint for trail management", Geneva, 03/99

42. http://www.infowin.org/ACTS/NEWS/CONTEXT UK/990899fr.htm "Invitation to the MEPHISTO public demonstration Marcoussis, France, 20-24 September 1999"

43. Bertelon, L. et al "OTN management interworking with SDH- Specifications, ACTS project no. AC209 Mephisto, deliverable D20 (restricted), the Mephisto Consortium, October 1999.

44. ITU-T draft rec. m.xxxc "Management services, object models and implementation ensembles for ATM-PON system", Geneva, January 2000.

45. Braden, R., et al "Resource ReSerVation Protocol (RSVP) - version 1 Functional Specification”, RFC 2205, September 1997

46. http://www.ietf.org/html.charters/intserv-charter.html

47. http://www.ietf.org/ids.by.wg/intserv.html

48. http://www.ietf.org/html.charters/diffserv-charter.html 
49. http://www.ietf.org/ids.by.wg/diffserv.html

50. http://www.ietf.org/html.charters/mpls-charter.htm

51. http://www.ietf.org/ids.by.wg/mpls.html

52. http://www.ietf.org/html.charters/rap-charter.html

53. http://www.ietf.org/html.charters/policy-charter.html

54 http://www.stardust.com/policy/whitepapers/qospol.htm 1994.

55. Anderson, J., et al "Protocols and architectures for IP optical networking", Bell Labs Technical Journal, January-March 1999

\section{Abbreviations}

ACTS: Advanced Telecommunication Technologies and Services

ASN.1: $\quad$ Abstract Syntax Notation no.1

ATM: Asynchronous Transfer Mode

AP: $\quad$ Access Point

BML: $\quad$ Business Management Layer

BVPN: Broadband Virtual Private Network

COPS: $\quad$ Common Open Policy Service

CP: $\quad$ Connection Point

DiffServ: Differentiated Services

CTP: $\quad$ Connection Termination Point

CORBA: Common Object Request Broker Architecture

CMIP: Common Management Information Protocol

cls: connectionless service

EML: Element Management Layer

ETSI: European Telecommunications Standards Institute

FEC: $\quad$ Forward Error Correction

FSAN: $\quad$ Full Service Access Network

FSN: $\quad$ Full Service Network

GNM: $\quad$ Generic Network Model

IDL: Interface Description Language

IETF: $\quad$ Internet Engineering Task Force

IntServ: Integrated Services

IP: Internet Protocol

ITU-T: International Telecommunication Union- Telecommunications sector

LC: $\quad$ Link Connection

LLA: $\quad$ Logical Layered Architecture

Mephisto: ManagemEnt of PHotonIc SysTems and netwOrks

MPLS: MultiProtocol Label Switching

NE: $\quad$ Network Element

NEF: $\quad$ Network Element Function

NML: $\quad$ Network Management Layer

OADM: Optical Add and Drop Multiplexer

OCH: Optical CHannel

OSF: $\quad$ Operation System Function

OTN: $\quad$ Optical Transport Network

PON: $\quad$ Passive Optical Network

QoS: $\quad$ Quality of Service 
RM-ODP: Reference Model -for Open Distributed Processing

RSVP: resource ReSerVation Protocol

SLA: $\quad$ Service Level Agreement

SLS: $\quad$ Service Level Specification

SML: $\quad$ Service Management Layer

SNC: $\quad$ SubNetwork Connection

TCP: Transport Connection Protocol

TMF: $\quad$ Tele Management Forum

TMN: Telecommunication Management Network

TTP: $\quad$ Trail Termination Point

UML: Unified Modeling Language

VCI: Virtual Container Identifier

VPI: Virtual Path Identifier

WDM: Wavelength Division Multiplex 